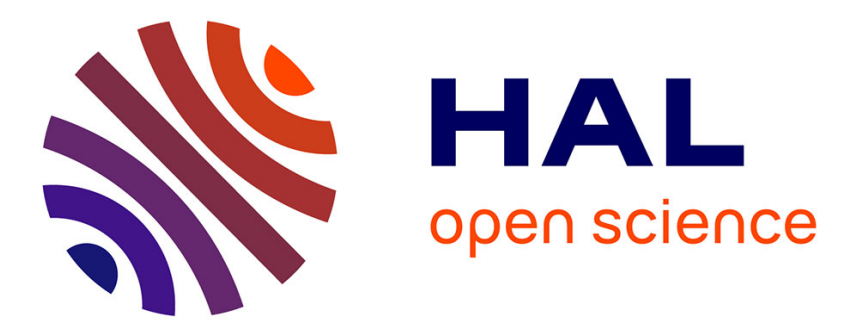

\title{
Single-Scan 13 C Diffusion-Ordered NMR Spectroscopy of DNP-Hyperpolarised Substrates
}

\author{
Ludmilla Guduff, Dennis Kurzbach, Carine Van Heijenoort, Daniel Abergel,
} Jean-Nicolas Dumez

\section{- To cite this version: \\ Ludmilla Guduff, Dennis Kurzbach, Carine Van Heijenoort, Daniel Abergel, Jean-Nicolas Dumez. Single-Scan 13 C Diffusion-Ordered NMR Spectroscopy of DNP-Hyperpolarised Substrates. Chemistry - A European Journal, 2017, 23 (66), pp.16722-16727. 10.1002/chem.201703300 . hal-03004240}

\author{
HAL Id: hal-03004240 \\ https://hal.science/hal-03004240
}

Submitted on 13 Nov 2020

HAL is a multi-disciplinary open access archive for the deposit and dissemination of scientific research documents, whether they are published or not. The documents may come from teaching and research institutions in France or abroad, or from public or private research centers.
L'archive ouverte pluridisciplinaire HAL, est destinée au dépôt et à la diffusion de documents scientifiques de niveau recherche, publiés ou non, émanant des établissements d'enseignement et de recherche français ou étrangers, des laboratoires publics ou privés. 


\title{
Single-scan ${ }^{13} \mathrm{C}$ diffusion-ordered NMR spectroscopy of DNP- hyperpolarised substrates
}

\author{
Ludmilla Guduff, ${ }^{[a]}$ Dennis Kurzbach, ${ }^{[b, c]}$ Carine van Heijenoort, ${ }^{[a]}$ Daniel Abergel ${ }^{[b, c]}$ and Jean-Nicolas \\ Dumez ${ }^{*[a]}$
}

\begin{abstract}
Diffusion-ordered NMR spectroscopy (DOSY) is a powerful approach for the analysis of molecular mixtures, yet its application range is limited by the relatively low sensitivity of NMR. We show here that spectrally resolved ${ }^{13} \mathrm{C}$ DOSY data can be collected, in a single scan, for substrates hyperpolarised by dissolution dynamic nuclear polarisation (D-DNP), which provides signal enhancements of several orders of magnitude. For this we use a convection-compensation pulse scheme, which we also analyse by numerical simulation. The proposed method further allows the acquisition of several consecutive DOSY spectra in a single D-DNP experiment.
\end{abstract}

The analysis of mixtures of small molecules is a frequently encountered and often challenging task in chemical science. In this respect, nuclear magnetic resonance (NMR) spectroscopy has many advantages, such as its non-destructive nature and the fact that it provides extensive structural information Diffusion-ordered spectroscopy (DOSY) is a particularly useful approach to analyse mixtures, ${ }^{[1]}$ in which NMR signals are separated according to the translational diffusion coefficients of the corresponding molecules. DOSY can be considered as a form of virtual chromatography, ${ }^{[2]}$ and provides information on molecular properties and interactions. ${ }^{[3]}$

The scope of analytical NMR methods, including DOSY, is limited by the relatively low sensitivity of NMR, which often prevents the analysis of low-concentrated and fast-changing mixtures. Several "hyperpolarisation" methods have been introduced, which address this sensitivity limitation. ${ }^{[4]}$ Dissolution dynamic nuclear polarisation (D-DNP) is a particularly powerful approach, which enables enhancements of up to 5 orders of magnitude in nuclear spin polarisation levels in the solution state. ${ }^{[4 \mathrm{a}]}$ The enhanced magnetisation, however, is only available for a limited time on the order of a few seconds, as determined by the longitudinal relaxation properties of the nuclear spins of interest. As a result, fast experiments are required to collect multidimensional data - corresponding in the case of DOSY to a pseudo-2D data set in which the diffusion attenuation is

[a] L. Guduff, Dr. C van Heijenoort, Dr. J.-N. Dumez Institut de Chimie des Substances Naturelles CNRS UPR2301, Univ. Paris Sud, Université Paris-Saclay 91190 Gif-sur-Yvette, France

E-mail: jeannicolas.dumez@cnrs.f

[b] Dr. D. Kurzbach, Dr. D. Abergel

Laboratoire des Biomolécules, Département de chimie

Ecole normale supérieure, UPMC Univ. Paris 06, CNRS, PSL

Research University

75005 Paris, France

[c] Dr. D. Kurzbach, Dr. D. Aberge

Laboratoire des Biomolécules

Sorbonne Universités, UPMC Univ. Paris 06, Ecole normale

supérieure, CNRS

75005 Paris, France

Supporting information for this article is given via a link at the end of the document incremented.

Among the methods that have been introduced for fast multidimensional NMR, the so-called "ultrafast" 2D NMR (UF NMR) approach is particularly well suited for D-DNP experiments. ${ }^{[5]}$ UF NMR relies on of the spatial encoding of NMR interactions and makes it possible to collect a $2 \mathrm{D}$ data set in a single scan, thus overcoming the time-constraints of D-DNP. UF 2D NMR has been combined with D-DNP for heteronuclear correlation experiments. ${ }^{[6]}$ Recent examples include the analysis of plant and cancer cell extracts. ${ }^{[7]}$ The principles of spatial encoding have been exploited to accelerate diffusion NMR experiments, ${ }^{[8]}$ and collect correlation maps of diffusion and relaxation properties from samples hyperpolarised with DDNP. ${ }^{[8]}$ A multi-scan diffusion MR approach has also been described for in vivo applications of D-DNP with slowly relaxing molecules. ${ }^{[9]}$ Other approaches to single-scan diffusion NMR experiments, such as Difftrain, ${ }^{[10]}$ have proven useful for the analysis of heterogeneous samples.

In this communication, we show that spectrally resolved ${ }^{13} \mathrm{C}$ DOSY data can be collected in a single scan from DNPhyperpolarised samples. Using a spin-echo-based spatially encoded approach, several DOSY data sets can be collected in a single scan. We also show that the use of a double-diffusionencoding (DDE) strategy is effective, in the spatial encoding case, to reduce the spurious effect of sample convection. This DDE approach, which we analyse with numerical simulation, is an efficient way to obtain good-quality DOSY spectra. The resulting protocol will make it possible to tap the sensitivity gains of D-DNP for DOSY-based analytical NMR applications.

Our experimental D-DNP approach is the following: ${ }^{[11]}$ the sample is mixed with radicals in a glass-forming mixture of water and glycerol (90\% deuteration), cooled to liquid-helium temperature, and irradiated with microwaves, to enhance the nuclear spin polarisation. Fast and efficient ${ }^{13} \mathrm{C}$ polarisation is achieved by performing a train of ${ }^{1} \mathrm{H}-{ }^{13} \mathrm{C}$ cross-polarisation (CP) sequences. ${ }^{[12]}$ After 30 mins of CP-based DNP, the sample is dissolved with $5 \mathrm{~mL}$ superheated $\mathrm{D}_{2} \mathrm{O}$ and transferred within $6 \mathrm{~s}$ via a magnetic tunnel to a high-resolution $800 \mathrm{MHz}$ NMR magnet for detection. These steps are summarised in Fig. 1a (see SI for details). In the solution state, the magnetisation decays irreversibly under the effect of nuclear spin relaxation and radio-frequency excitation pulses. As a result, most multidimensional experiments must be adapted when used in combination with D-DNP.

The acquisition of spectrally resolved DOSY data in a single scan can be achieved by a spatial parallelization, in which different virtual slices receive different diffusional attenuations. ${ }^{[8 a]}$ In practice, the diffusion delay is preceded (or followed) by a combination of magnetic-field gradients and frequency swept pulses that winds (or unwinds) a spatially quadratic phase. This scheme results in a spatially dependent attenuation, which can be measured in a spectrally resolved manner with echo planar spectroscopic imaging. To extract the diffusion coefficients, $D$, 
from the data, the spatial profile for each resonance, $S(z)$, is fitted to a modified Stejskal-Tanner equation

$$
S(z)=S_{0} \exp \left(-D \Delta(K(z))^{2}\right)
$$

where $K(z)$ is the derivative with respect to position of the spin phase imparted by the chirp and gradient pair used for spatial encoding, ${ }^{[8 \mathrm{~d}]}$ and $\Delta^{\prime}$ is an effective diffusion time. Several pulse sequences have been reported for such spatially encoded diffusion-ordered NMR spectroscopy (SPEN DOSY) experiments, including stimulated-echo and spin-echo-based experiments. ${ }^{[8,13]}$ Here, a spin-echo based sequence is selected to preserve magnetisation, both within a scan (STE-based sequences sacrifice half or more of the magnetisation) and from scan to scan. Figure $1 \mathrm{~b}$ shows an initial spin-echo SPEN DOSY pulse sequence, adapted from Ref. ${ }^{[b]}$ (where a similar scheme is shown, but not used). One limitation of spin-echo based DOSY pulse sequences is the possibly strong modulation of signals by $\mathrm{J}$-couplings during the long diffusion delay. For the sample analysed here, no such J-modulation is present. Magnitude processing of the data, which is common in spatially encoded NMR, would be used in other cases; this would lead to J-modulated amplitudes.

(a)

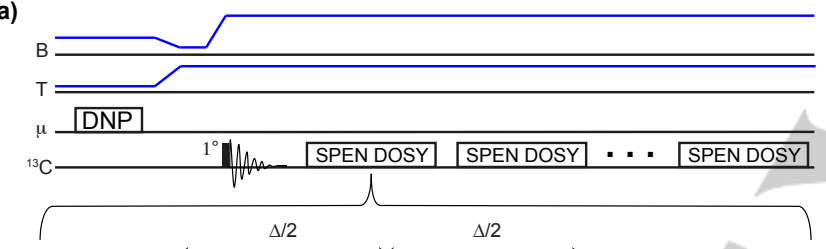

(b)
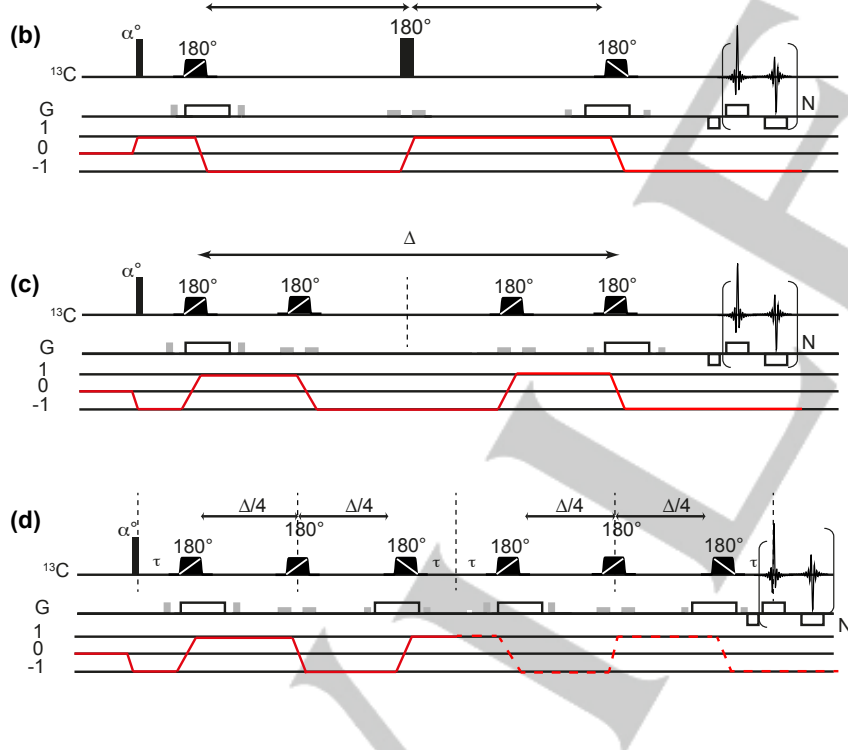

Figure 1. Description of the D-DNP SPEN DOSY experiment. a) Timing of the polarisation, dissolution and acquisition steps, with the evolution of the temperature and magnetic field. b) Spin-echo SPEN DOSY pulse sequence. c) Double spin-echo SPEN DOSY pulse sequence. d) Double diffusion encoding SPEN DOSY pulse sequence. Crusher gradients are shown in grey. The selected coherence transfer pathway is shown in red.

The non-renewable nature of the hyperpolarisation generated with D-DNP imposes further constraints on the experimental design, if a time-series of spectra is to be recorded rather than a single one. This is particularly important for the applications of D-DNP to the monitoring of chemical and biochemical reactions. ${ }^{[14]}$ Figure $1 \mathrm{c}$ shows a double-spin-echo SPEN DOSY pulse sequence that uses adiabatic pulses for refocusing. When a small-tip-angle excitation pulse is used, the residual magnetisation is restored by the even number of refocusing pulses and preserved for subsequent scans. In addition, adiabatic pulses are more robust against RF inhomogeneities, and have negligible effect on the signal's phase when used in pairs. This approach is inspired by fast spectroscopic imaging experiments. ${ }^{[15]}$

For our first demonstration of the hyperpolarised SPEN DOSY experiment, we selected three small molecules that are commonly used in D-DNP. The analysed sample consisted of a mixture of acetate, pyruvate and urea, each containing a ${ }^{13} \mathrm{C}$ labelled quaternary carbon. Since D-DNP efficiently polarises a large range of substrates, the resulting protocol is broadly applicable.

An important challenge in diffusion NMR experiments is to encode the microscopic Brownian motion of interest, while global displacements take place on much larger scales. In this respect, DOSY NMR is known to be particularly sensitive to convection effects. ${ }^{[16]}$ In D-DNP experiments, motions other than diffusion are expected after injection, which may affect the diffusion measurement. This is indeed observed in a time-series of DOSY spectra obtained in a single D-DNP experiment, shown in Fig. 2a-c. During the detection period after dissolution, SPEN DOSY was carried out in intervals of $10 \mathrm{~s}$ after dissolution with detection angles of $30^{\circ}$ (chosen as an empirical compromise between the number of scans that can be acquired and the sensitivity per scan). The spatial profiles, shown in Fig. 2d-f display artefactual oscillations and accentuated decays that prevent the determination of diffusion coefficients and the separation of the mixtures' components.

In classic diffusion NMR experiments, the effect of sample convection can be significantly reduced with the use of a double rather than single diffusion-encoding step. In this approach, introduced by Jerschow and Müller, ${ }^{[17]}$ the phase imparted by flow effects changes sign from one diffusion-encoding step to the other, whereas the effect of diffusion is cumulative. Robust diffusion data can be recovered, e.g., in solvents that are prone to strong convection effects. Such a double-diffusion-encoding (DDE) SPEN DOSY pulse sequence is shown in Fig. 1d (the name is borrowed form microstructure studies). ${ }^{[18]}$ The coherence transfer pathway leads to a cancellation of the flowinduced phase shift at the end of the second encoding step.

Figure 2g-i shows a time series of DOSY NMR spectra recorded with a double-diffusion encoding SPEN DOSY experiment. With the DDE approach, the diffusion decay curves, shown in Fig. 2j-I have the expected qualitative behaviour. The DOSY display of the data highlights the good separation of urea from pyruvate and acetate. Importantly, the fitted diffusion coefficients are found to vary by less than $7 \%$ over the three consecutive scans (see SI). Overall, the DDE SPEN DOSY experiment provides a robust approach to make use of hyperpolarised ${ }^{13} \mathrm{C}$ signals for diffusion experiments. For systems with possibly time-dependent composition, e.g., because of chemical reactions or of molecular interactions, the 

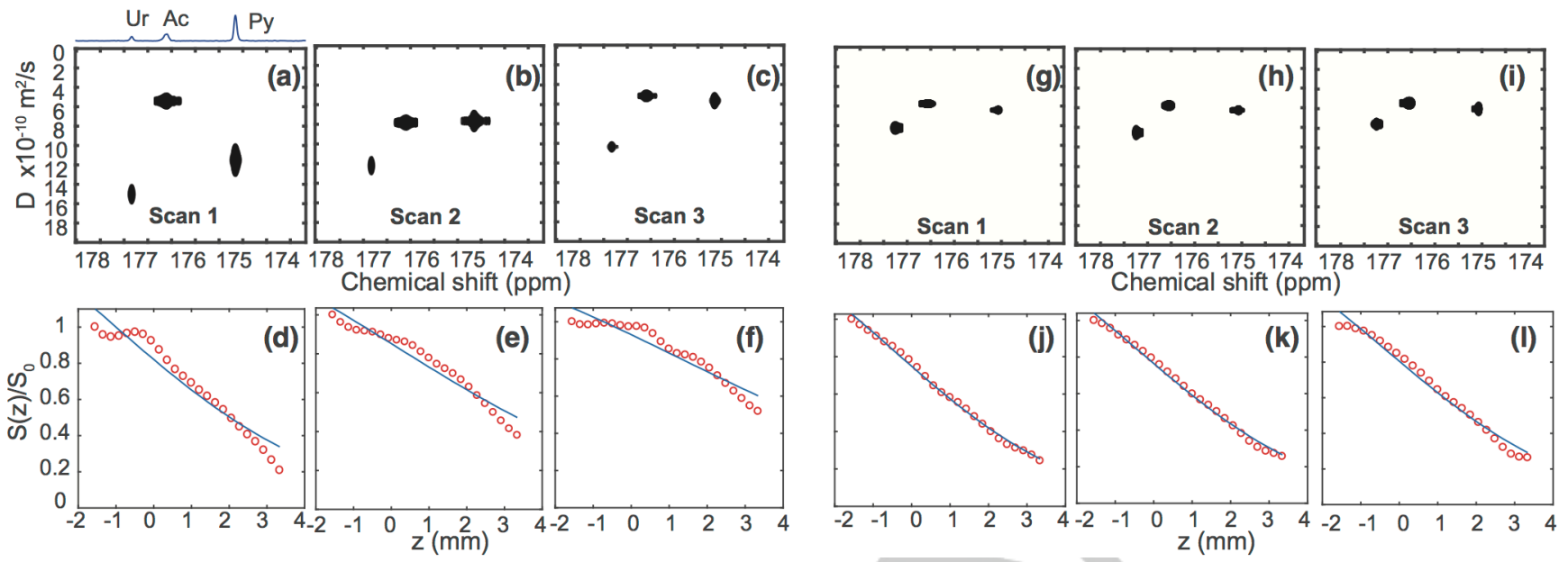

Figure 2: Time series of DOSY data obtained on a mixture of pyruvate, acetate and urea. a-c) DOSY spectra for the double-spin-echo SPEN DOSY pulse sequence, obtained in a single D-DNP experiment; d-f) associated fitted diffusion curves for pyruvate; g-i) DOSY spectra for the double-diffusion-encoding SPEN DOSY pulse sequence, obtained in a single D-DNP experiment; $j-l$ ) associated fitted diffusion curves for pyruvate. The peaks are folded within the reduced spectral width of the SPEN DOSY experiment (see SI). The detection was carried out with a $800 \mathrm{MHz}$ spectrometer.

The outcome of the double-diffusion-encoding approach may be further rationalised by numerical simulation of the spatially encoded experiments in the presence of flow. We, with the Kuprov group, have recently reported the efficient simulation of SPEN DOSY experiments, using a Fokker-Planck description of the spin and space dynamics. ${ }^{[8 d, 19]}$ The effect of a bulk displacement of the spins can be included in these simulations, by adding a "hydrodynamics" component to the evolution operator in the equation of motion. ${ }^{[20]}$ Figure $3 a$ and $3 b$ show the comparison of single (SDE) and double diffusion encoding experiments. In the absence of flow, the two pulse sequences yield identical results. When the spins undergo a bulk motion, however, the spatial profile in the SDE case is severely affected, with a faster decay and oscillations that are qualitatively similar to the experimental results shown in Fig. $3 \mathrm{c}$ and Fig 2a-c. In contrast, the spatial profile obtained with the DDE pulse sequence is much less affected by flow. The flow is defined here as linear, constant, and in the direction of the encoding magnetic field gradient. For an input diffusion value of $8 \times 10^{-10} \mathrm{~m}^{2} . \mathrm{s}^{-1}$ and a flow value of $12 \mathrm{~mm} . \mathrm{s}^{-1}$, a fit of the diffusion decay curve yields $\mathrm{D}=7.11 \times 10^{-10} \mathrm{~m}^{2} \cdot \mathrm{s}^{-1}$ which differs by less than $12 \%$. The present simulation captures the concept of the DDE approach. Simulations with more realistic velocity distributions will be addressed in future studies.

A further benefit of these numerical simulations is the possibility to design an improved model to fit the experimental data. Specifically, the effect of the finite width of the encoding and decoding gradients can be accounted for by considering an effective diffusion delay $\Delta^{\prime}$, which is slightly different from the nominal delay $\Delta$. For the spin-echo pulse sequence shown in Fig. $1 \mathrm{~d}$, the encoding and decoding chirp pulses have opposite sweep rates. As a result, the effective diffusion delay is positiondependent. A simple expression:

$$
\Delta^{\prime}=\Delta+4 T_{e}\left(\frac{z}{L}-1\right)
$$

where $T_{e}$ is the duration of the chirp pulse and $L$ is the length of the region swept by the chirp pulse, is obtained by analysing numerical simulations. Using this corrected diffusion delay yields a more accurate value of the diffusion coefficient.

Both conventional and spatially encoded DOSY experiments are known to be afflicted by a variety of systematic errors that originate from temperature effects, ${ }^{[16]}$ magnetic-field gradient non-linearity, ${ }^{[21]}$ etc. Here, the diffusion coefficients obtained with the hyperpolarised experiments differ from reference values obtained with a conventional, thermal-equilibrium experiment by about $15 \%$ (see supporting information). Further investigations are in progress to characterise the sources of this discrepancy, which likely includes temperature and gradient effects. Importantly, systematic errors do not prevent the separation of components in a mixture, or the characterisation of interactions, provided that suitable calibration procedures are used. ${ }^{[3 c]}$ While the limited spectral width accessible with EPSI results in peak folding, peak overlapping could be avoided with spectrally selective pulses or "interleaved" acquisitions. ${ }^{[22]}$

D-DNP provides signal enhancements of several orders of magnitude for high-resolution solution-state NMR. Using a magnetic field of $18.8 \mathrm{~T}$ for detection (corresponding to a ${ }^{1} \mathrm{H}$ Larmor frequency of $800 \mathrm{MHz}$ ), signal enhancements of the order of 10000 are routinely obtained for ${ }^{13} \mathrm{C}$ with our setup. Because of the relatively long transfer time, as well as the time allocated here to record a $1 \mathrm{D}{ }^{13} \mathrm{C}$ spectrum, the signal enhancements obtained for the SPEN DOSY data shown in Fig. $2 \mathrm{~g}$ was found to be of about 1800 , as measured for pyruvate. Higher enhancements are expected with the use of faster transfer systems, which will also make it possible to observe faster-relaxing nuclei and in particular non-quaternary carbons. ${ }^{[23]}$ The actual limit of detection will also depend on 
other factors such as sample dilution, and the removal or conservation of polarisation agents. Other hyperpolarisation methods, such as SABRE that has recently been used for multiscan DOSY analyses, ${ }^{[24]}$ should also be compatible with singlescan acquisitions.
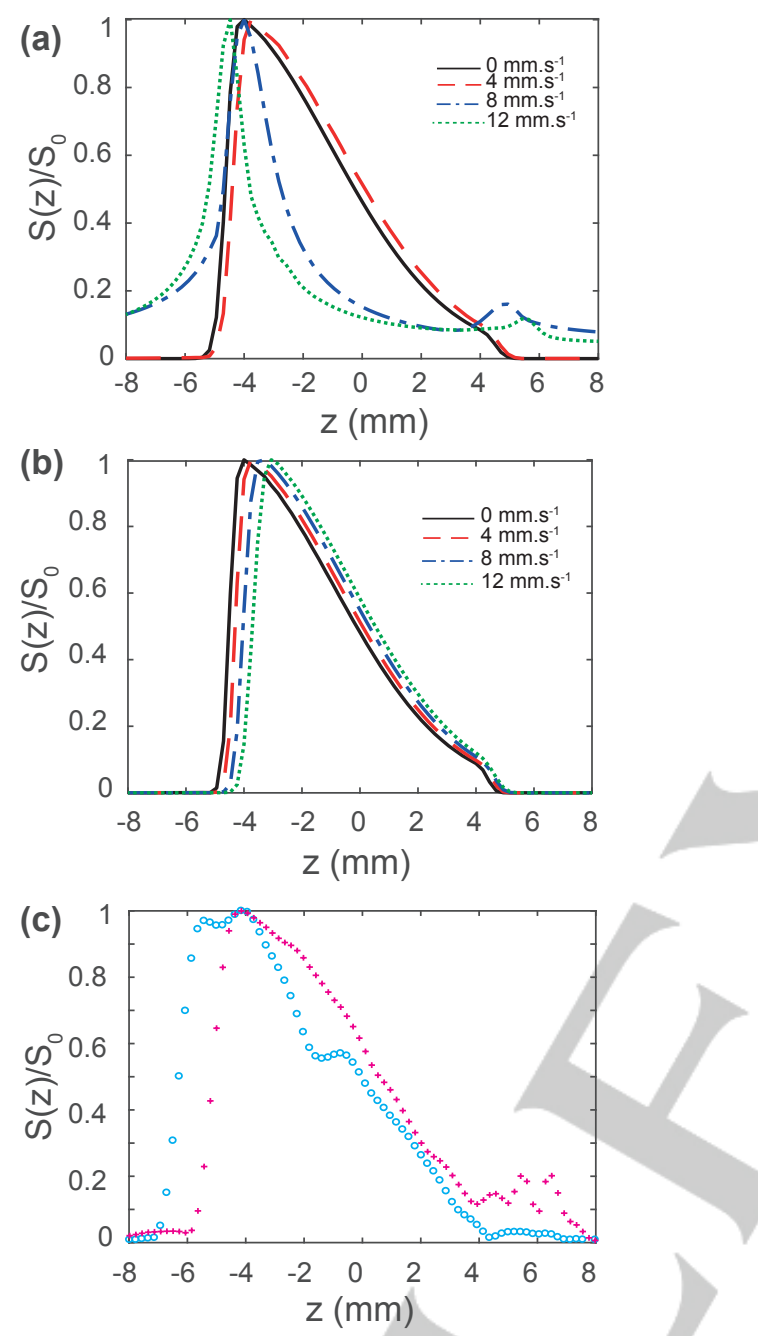

Figure 3. Numerical simulation of the effect of flow for SPEN DOSY pulse sequences a) simulation results of double-spin-echo SPEN DOSY pulse sequence for several flow values b) Simulation results of double-diffusionencoding SPEN DOSY pulse sequence for several flow values. c) Experimentally measured spatial profile for pyruvate for the SDE (blue) and DDE (red) pulse sequence.

In summary, we have shown that DOSY NMR spectra can be collected in a single scan from samples hyperpolarised with D-DNP, using an acquisition strategy based on spatial encoding. The use of a double rather than a single diffusion-encoding step makes it possible to attenuate convection effects, which is also supported by numerical simulations. The resulting approach will broaden the range of mixtures that may be analysed with diffusion NMR as it renders not only carbon-direct detection in combination with DOSY possible, but also the time-resolved monitoring of diffusion properties.

\section{Experimental Section}

Full experimental details are given in the supporting information

\section{Acknowledgements}

The authors thank Prof. Geoffrey Bodenhausen for insightful discussions, as well as Bruker BioSpin for providing the dissolution-DNP setup. This research was supported by the Région Ile-de-France, the Agence Nationale de la Recherche (ANR-16-CE29-0012) and the European Research Council (ERC, contract "dilute para-water"). Financial support from the TGIR-RMN-THC Fr3050 CNRS for conducting the research is gratefully acknowledged.

[1] a) C. S. Johnson, Prog. Nucl. Magn. Reson. Spectrosc. 1999, 34, 203-256; b) H. Barjat, G. A. Morris, S. Smart, A. G. Swanson and S. C. R. Williams, J. Magn. Reson., Ser B 1995, 108, 170-172.

[2] a) A. A. Colbourne, G. A. Morris and M. Nilsson, J. Am. Chem. Soc. 2011, 133; b) I. Toumi, B. Torresani and S. Caldarelli, Anal. Chem. 2013, 85, 11344 11351 ; c) A. A. Colbourne, S. Meier, G. A. Morris and M. Nilsson, Chem. Commun. 2013, 49, 10510-10512.

[3] a) R. Neufeld and D. Stalke, Chemical Science 2015, 6, 3354-3364; b) R. Neufeld, T. L. Teuteberg, R. Herbst-Irmer, R. A. Mata and D. Stalke, J. Am. Chem. Soc. 2016, 138, 4796-4806; c) D. Li, I. Keresztes, R. Hopson and P. G. Williard, Acc. Chem. Res. 2009, 42, 270-280; d) S. Bachmann, B. Gernert and D. Stalke, Chem. Commun. 2016, 52, 12861-12864.

[4] a) J. H. Ardenkjaer-Larsen, B. Fridlund, A. Gram, G. Hansson, L. Hansson, M. H. Lerche, R. Servin, M. Thaning and K. Golman, Proc. Natl. Acad. Sci. U.S.A. 2003, 100; b) R. W. Adams, J. A. Aguilar, K. D. Atkinson, M. J. Cowley, P. I. P. Elliott, S. B. Duckett, G. G. R. Green, I. G. Khazal, J. Lopez-Serrano and D. C. Williamson, Science 2009, 323, 1708; c) C. R. Bowers and D. P. Weitekamp, J. Am. Chem. Soc. 1987, 109, 5541-5542.

[5] a) L. Frydman, T. Scherf and A. Lupulescu, Proc. Natl. Acad. Sci. U.S.A. 2002, 99, 15858-15862; b) P. Giraudeau and L. Frydman, Annu. Rev. Anal. Chem. 2014, 7, 129-161.

[6] a) L. Frydman and D. Blazina, Nature Physics 2007, 3, 415-419; b) P. Giraudeau, Y. Shrot and L. Frydman, J. Am. Chem. Soc. 2009, 131, 13902-+.

[7] J.-N. Dumez, J. Milani, B. Vuichoud, A. Bornet, J. Lalande-Martin, I. Tea, M. Yon, M. Maucourt, C. Deborde, A. Moing, L. Frydman, G. Bodenhausen, S. Jannin and P. Giraudeau, Analyst 2015, 140, 5860-5863.

[8] a) M. J. Thrippleton, N. M. Loening and J. Keeler, Magn. Reson. Chem. 2003, 41, 441-447; b) Y. Shrot and L. Frydman, J. Magn. Reson. 2008, 195, 226-231; c) S. Ahola, V. V. Zhivonitko, O. Mankinen, G. Zhang, A. M. Kantola, H.-Y. Chen, C. Hilty, I. V. Koptyug and V.-V. Telkki, Nature Communications 2015, 6, 8363; d) L. Guduff, I. Kuprov, C. van Heijenoort and J.-N. Dumez, Chem. Commun. 2017, 53, 701-704.

[9] B. L. Koelsch, K. R. Keshari, T. H. Peeters, P. E. Z. Larson, D. M. Wilson and J. Kurhanewicz, Analyst 2013, 138, 1011-1014.

[10] J. P. Stamps, B. Ottink, J. M. Visser, J. P. M. van Duynhoven and R. Hulst, J. Magn. Reson. 2001, 151, 28-31.

[11] D. Kurzbach, E. M. M. Weber, A. Jhajharia, S. F. Cousin, A. Sadet, S Marhabaie, E. Canet, N. Birlirakis, J. Milani, S. Jannin, D. Eshchenko, A Hassan, R. Melzi, S. Luetolf, M. Sacher, M. Rossire, J. Kempf, J. A. B. Lohman, M. Weller, G. Bodenhausen and D. Abergel, J. Chem. Phys. 2016 , 145, 194203.

[12] A. Bornet, R. Melzi, A. J. P. Linde, P. Hautle, B. van den Brandt, S. Jannin and G. Bodenhausen, Journal of Physical Chemistry Letters 2013, 4, 111-114. [13] S. Ahola, O. Mankinen and V. V. Telkki, Magn. Reson. Chem. 2017, 55, 341-347.

[14] a) S. Bowen and C. Hilty, Angew. Chem. Int. Ed. 2008, 47, 5235-5237; b) E. Miclet, D. Abergel, A. Bornet, J. Milani, S. Jannin and G. Bodenhausen, Journal of Physical Chemistry Letters 2014, 5, 3290-3295.

[15] C. H. Cunningham, A. P. Chen, M. J. Albers, J. Kurhanewicz, R. E. Hurd, Y. F. Yen, J. M. Pauly, S. J. Nelson and D. B. Vigneron, J. Magn. Reson. 2007 187, 357-362

[16] I. Swan, M. Reid, P. W. A. Howe, M. A. Connell, M. Nilsson, M. A. Moore and G. A. Morris, J. Magn. Reson. 2015, 252, 120-129.

[17] A. Jerschow and N. Muller, J. Magn. Reson. 1997, 125, 372-375.

[18] N. Shemesh, S. N. Jespersen, D. C. Alexander, Y. Cohen, I. Drobnjak, T. B. Dyrby, J. Finsterbusch, M. A. Koch, T. Kuder, F. Laun, M. Lawrenz, H. Lundell, P. P. Mitra, M. Nilsson, E. Ozarslan, D. Topgaard and C. F. Westin, Magnetic Resonance in Medicine 2016, 75, 82-87. 
[19] L. Guduff, A. J. Allami, C. van Heijenoort, J.-N. Dumez and I. Kuprov, Phys. Chem. Chem. Phys. 2017.

[20] I. Kuprov, J. Magn. Reson. 2016, 270, 124-135.

[21] M. A. Connell, P. J. Bowyer, P. A. Bone, A. L. Davis, A. G. Swanson, M. Nilsson and G. A. Morris, J. Magn. Reson. 2009, 198, 121-131.
[22] L. Frydman, A. Lupulescu and T. Scherf, J. Am. Chem. Soc. 2003, 125, 9204-9217.

[23] H.-Y. Chen and C. Hilty, Chemphyschem 2015, 16, 2646-2652.

[24] I. Reile, R. L. E. G. Aspers, J.-M. Tyburn, J. G. Kempf, M. C. Feiters, F. P.

J. T. Rutjes and M. Tessari, Angew. Chem. Int. Ed. n/a-n/a. 


\section{Entry for the Table of Contents}

\section{COMMUNICATION}

${ }^{13} \mathrm{C}$ DOSY NMR spectra are obtained in a single scan from DNP-hyperpolarised substrates.

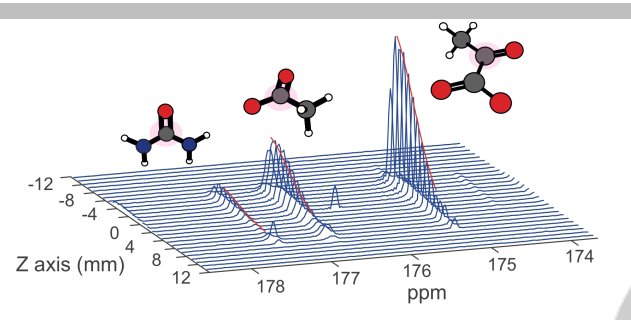

Author(s), Corresponding Author(s)*

Page No. - Page No.

Title 